\title{
Sketching-out Virtual Humans: A Smart Interface for Human Modelling and Animation
}

\author{
Chen $\mathrm{Mao}^{1}$, Sheng Feng Qin ${ }^{1}$, and David Wright ${ }^{1}$, \\ ${ }^{1}$ School of Engineering and Design, Brunel University, Uxbridge, UB8 3PH, UK \\ \{Chen.Mao, Sheng.Feng.Qin, David.Wright\}@ brunel.ac.uk
}

\begin{abstract}
In this paper, we present a fast and intuitive interface for sketching out $3 \mathrm{D}$ virtual humans and animation. The user draws stick figure key frames first and chooses one for "fleshing-out" with freehand body contours. The system automatically constructs a plausible 3D skin surface from the rendered figure, and maps it onto the posed stick figures to produce the $3 \mathrm{D}$ character animation. A "creative model-based method" is developed, which performs a human perception process to generate $3 \mathrm{D}$ human bodies of various body sizes, shapes and fat distributions. In this approach, an anatomical 3D generic model has been created with three distinct layers: skeleton, fat tissue, and skin. It can be transformed sequentially through rigid morphing, fatness morphing, and surface fitting to match the original $2 \mathrm{D}$ sketch. An auto-beautification function is also offered to regularise the $3 \mathrm{D}$ asymmetrical bodies from users' imperfect figure sketches. Our current system delivers character animation in various forms, including articulated figure animation, 3D mesh model animation, 2D contour figure animation, and even 2D NPR animation with personalised drawing styles. The system has been formally tested by various users on Tablet PC. After minimal training, even a beginner can create vivid virtual humans and animate them within minutes.
\end{abstract}

Keywords: sketching interface, human modelling and animation, creative model-based method, multi-layered generic model, morphing.

\section{Introduction}

Research in human modelling and animation has involved effort over many decades. Nowadays, their application has penetrated a great variety of fields, including industry, military, biomedicine, and education. In today's public entertainment, virtual humans are playing a particularly remarkable role when engaged in 3D games, Hollywood films, and multimedia (virtual human presenter).

Current virtual human generation methods can be classified into three major categories: creative [1][2], reconstructive [3][4], and interpolated [5][6]. Although these approaches are capable of creating highly realistic human body models with various appearances and motions, they require extensive expertise, special equipment (traditional/video camera, 3D body scanner, motion capture system, etc), and proficient computer skills. Therefore, it is extremely difficult for regular users (2D artists/designers and ordinary users) to participate and create their own 3D virtual human models. 
Sketching, however, is probably one of the most popular and approachable ways to quickly rough out characters and their motions. Since ancient times, from the proficient to novice, people have, with ease and familiarity, drawn their imaginary or real characters with pencil and paper. Hence, developing a sketch-based tool for generating graphics and enabling the use experiences is crucial. It can also transfer ordinary users into the 3D animation world via their favoured medium - sketching.

Regarding sketch-based human modelling and animation, two categories of related work can be basically identified: sketch-based $3 D$ freeform modelling and sketchbased character animation. The former research category represented by [7][8] transfers users' 2D freehand drawings into 3D freeform surfaces of stuffed toys, simple clothes, car/furniture models, etc, in various ways. However, sketch-based 3D human body modelling has remained a difficult undertaking, which has rarely been addressed before. Since the human body has an irregular and complex surface and our eyes are especially sensitive to the human figure, current modelling techniques constrained by their underlying construction schemes, are inadequate for obtaining plausible results. Moreover, these interfaces accept mainly single and clean stroke input and enforce a "part-by-part" drawing routine, where one sketch stroke often specifies the shape of an entire object part and modelling an object is more like assembling a handcraft toy than real freehand sketching.

The current research on sketch-based character animation is demonstrated by various sketching interfaces for articulated figure animation [9][10], motion doodling [11], cartoon storyboarding [12], etc. Although impressive, many of these systems deal with simple characters, rather than sophisticated human characters with realistic and various appearances. Moreover, none of them has delivered a complete picture of "sketch-based modelling and animation", including key framing, figure pose recognition, 2D-3D surface modelling, and the resulting animations performed by various levels of characters.

In this paper, we present our new method and a novel sketching interface, which enable anyone who can draw to "sketch-out" 3D virtual humans and animation. Our sketching interface can automatically recognise users' 2D figure sketches into 3D human bodies of various body sizes, shapes and fat distributions. It supports a natural drawing process and allows rough and sketchy 2D figure input (with multi-stroke profiles, missing body contours, and drawing imperfections). Users can incrementally modify their 3D models by over-sketching 2D figure. The resulting animations can be delivered in various forms, including articulated figure animation, 3D mesh model animation, 2D contour figure animation, and even 2D NPR animation with personalised drawing styles. This smart interface enriches the user experiences.

Figure 1 illustrates the graphical pipeline of this virtual human sketching interface. Users first draw stick figure key frames to define a specific motion. Then, they can "flesh-out" any existing stick figure with body profiles to portray an imaginative character. The system can automatically "perceive" the body size (skeleton proportion) and shape (body profile and fat distribution) from the sketched figure, and recongise it into a 3D virtual character through continuous graphical comparisons and generic model morphing (rigid morphing, fatness morphing, and surface fitting). Our anatomical generic model is created from Visible Human cross-section images [13], which are encapsulated with three distinct layers: skeleton, fat tissue, and skin for undertaking both geometric and physical morphing. The resulting 3D skin surface can 
be mapped onto each of the posed stick figure key frames [16], which can be further interpolated as final 2D/3D animations. Here, the $3 \mathrm{D}$ poses are reconstructed from $2 \mathrm{D}$ stick figure sketches through a "multi-layered back-front ambiguity clarifier" [10].
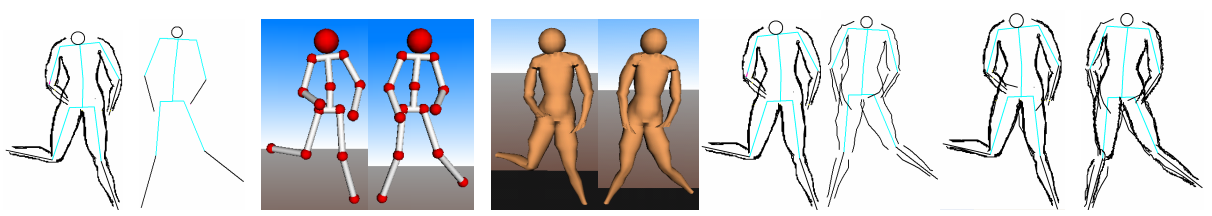

Fig. 1. Sketching out a virtual human and animating it in both $2 \mathrm{D}$ and $3 \mathrm{D}$ world.

\section{Sketch-based Human Modelling and Animation Pipeline}

As in [14], humans are accustomed to doing the reverse projection of sketched geometry from $2 \mathrm{D}$ back into $3 \mathrm{D}$. It is, however, mathematically indeterminate and very difficult to emulate computationally. To decompose the complexity of direct 3D modelling and animation from 'noisy' figure sketches (featured by foreshortening, contour over-tracing, body part overlapping, shading/shadow, etc.), we designed a "Stick Figure [10] $\rightarrow$ Fleshing-out $\rightarrow$ Skin Mapping" pipeline (described in Section 1). This is inspired by the drawing sequence recommended by many sketch books. In principle, it echoes the modelling and animation pipeline in commercial packages (e.g. 3DS MAX, Maya). Regarding our current design, functionalities at different levels are gained for different users. Thus they can choose to make simple stick figures, create delicate 3D surface models, or explore further to animate these sketchgenerated creatures. Moreover, models can be exported to commercial packages at any level to be refined by their function kits.

\section{Creative Model-based 3D Body Generation Scheme}

As discussed earlier, humans are capable of instinctively perceiving a 'noisy' 2D figure sketch as a realistic 3D body. Thus, understanding this perception process is essential for realising the automatic 2D-to-3D reconstruction. Since we see and interact with people everyday, our brain has been familiarised with various body shapes and the correlations between 2D flat features and their real 3D counterparts [14]. Therefore, when observing a raw figure sketch, our brain can automatically clean-up the distracting 'noises', perceive the body size and shape, recall an associated body shape from memory, and then morph it and fit it into the 2D drawing to obtain the final 3D image. Theoretically, if given a range of pre-stored morphable template bodies, a computer is able to perform this as well, through performing the similar 'thinking', 'recalling', and morphing routines.

In terms of the $3 \mathrm{D}$ body templates, the statistically parameterised models generated from [5][6] may provide a rich resource of realistic human body shapes. However, each figure sketch depicts an imaginative character, which has a unique and even 
unrealistic body shape that often blends the features of many different bodies, together with its individualised attributes. Thus, it is very hard to find a matching model from the existing body geometries through a fixed number of sizing parameters. Since the human brain is able to morph the body shape both geometrically and physically to fit-in the 2D sketch, it is necessary to introduce more physical layers (i.e. fat, muscle) into the current two-layered (skeleton-skin) digital template [5][6], to match it more flexibly into the 2D drawing with plausible effects. In our system, we adopted a three-layered (skeleton-fat tissue-skin) anatomical model, because compared with muscle, the fat condition is more variable and dominant in affecting the overall body shape.

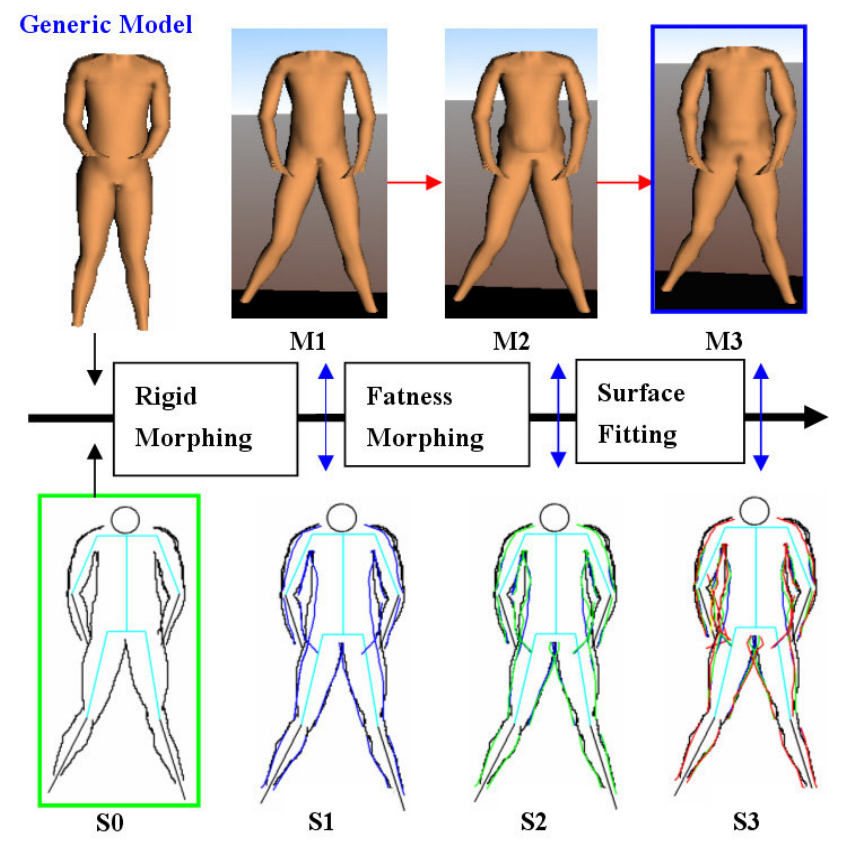

Fig. 2. Transfer a 2D freehand sketch into the 3D body model through an automatic perception and morphing process: Users draw a $2 \mathrm{D}$ figure (S0). The system automatically retrieves its $3 \mathrm{D}$ pose and body proportion, and performs a rigid morphing on the 3D generic model. The resulting 3D model $\mathrm{M} 1$ is projected into $2 \mathrm{D}$ (blue lines in $\mathrm{S} 1$ ) and compared with the original sketch (black lines) to evaluate its body fat distribution. M1 is then deformed through fatness morphing into M2, which is projected (green lines in S2) and compared again with the 2D sketch to get the fitting measurements. The final 3D model (M3) is delivered to users, after an automatic surface fitting and beautification process (on M2). Users can incrementally refine their 2D sketches; a similar perception process is performed to update the $3 \mathrm{D}$ model.

We investigated a "Creative Model-based Method", which can perceive the size and shape of a sketched figure and recognise it into a plausible 3D counterpart model, through continuous graphical comparisons and template morphing (see Figure 2). Our 3-layered generic model is digitised from the anatomical male cross-section images of The Visible Human Project ${ }^{\circledR}$ [13]. Although only one template model is employed in the current system, our generic model acquisition technique can be easily replicated 
on the CT cross-section images generated from whole body CT scanning equipment [15], to create a population of layered generic models of males, females, children, and elderly people for modelling purposes. Since each model is morphable in both geometric and physical senses, it can generate a variety of new body shapes on its own to further expand the template database and enrich virtual human population. After obtaining an initial 3D model from the 2D sketch, incremental modification can be made to change the global body shape. Since a "Stick Figure $\rightarrow$ Fleshing out" routine is followed, this sketch-generated model is readily animatable. It inherits the skin binding conditions ${ }^{1}$ of the 3D generic model. Our current system cannot support sketch-based modelling of human head/hands/feet, which is also a common challenge for other related approaches.

\section{Generic Model Acquisition and Specification}

The generic model in our system is reconstructed from cadaveric cross-section images from the Visible Human Project ${ }^{\circledR}$ of the National Library of Medicine [13]. The preparation of this multi-layered anatomical model entails: Virtual Skeleton Registration, Skin Mesh Recovery, Template Fat Distribution Digitisation, and Template Fat Percentage Manipulation.

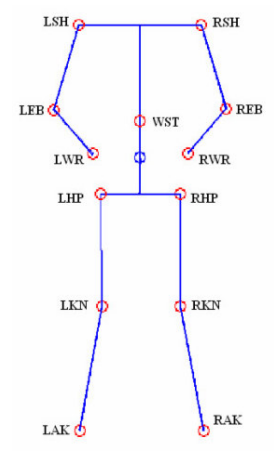

(a)

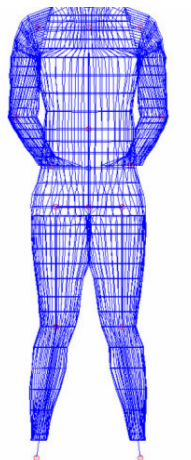

(b)

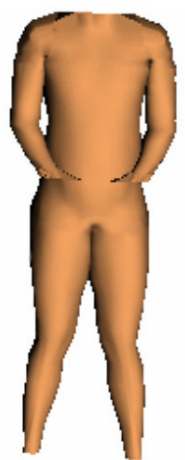

(c)

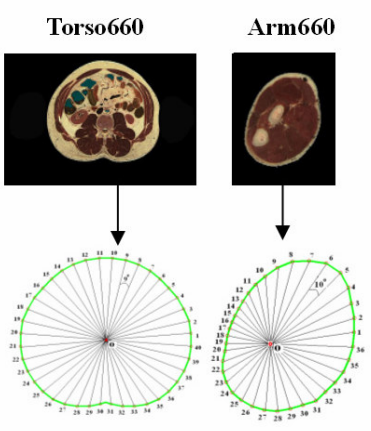

(d)

Fig. 3. (a) The template skeleton (joint -red circle, body root-blue circle). (b) The wireframe 3D generic model. (c) The rendered 3D generic model (d) Ray casting the cross section images for each body part (torso-40 rays, limbs-36 rays) to digitise the $3 \mathrm{D}$ template from the human cadaveric images. The ray centre $\mathrm{O}$ is the estimated sectional bone position. The image index indicates the corresponding body part and the distance from the head top to the give cross-section slice.

\subsection{Virtual Skeleton Registration \& Skin Mesh Recovery}

To apply our simplified skeleton model [10] to the anatomical human body, we used a semi-automatic image registration method. After the skeleton adaptation, the extracted

1 In the multi-layered generic model, the skin is bound to the underlying skeleton through assigning the affecting bones and corresponding weights for each mesh vertex. 
bone/joint measurements are further normalised to achieve the template skeleton, which conforms to the system's pre-stored reference skeletons. To digitise the skin mesh, we divide the overall body into individual parts, and perform "ray casting" (see Figure 3(d)) for each body part on their indexed/calibrated cross-section images. The ray centres here are acquired from linear interpolation of the neighbouring template joints. The 3D template mesh is constructed by connecting all 3D casting points horizontally and vertically, which results in a wire frame model with 3136 vertices (see Figure 3(b)). This quadpatch grid structure enables easy feature extraction from raw figure sketches, and the piecewise affine transformation of the $3 \mathrm{D}$ template.

\subsection{Template Fat Distribution Digitisation}

Instead of a general fat distribution [6] describing typical body shapes (i.e. apple, pear), we developed a comprehensive distribution map (see Figure 4), comprising the fat accumulating condition for each body part, each cross section, and each skin point.
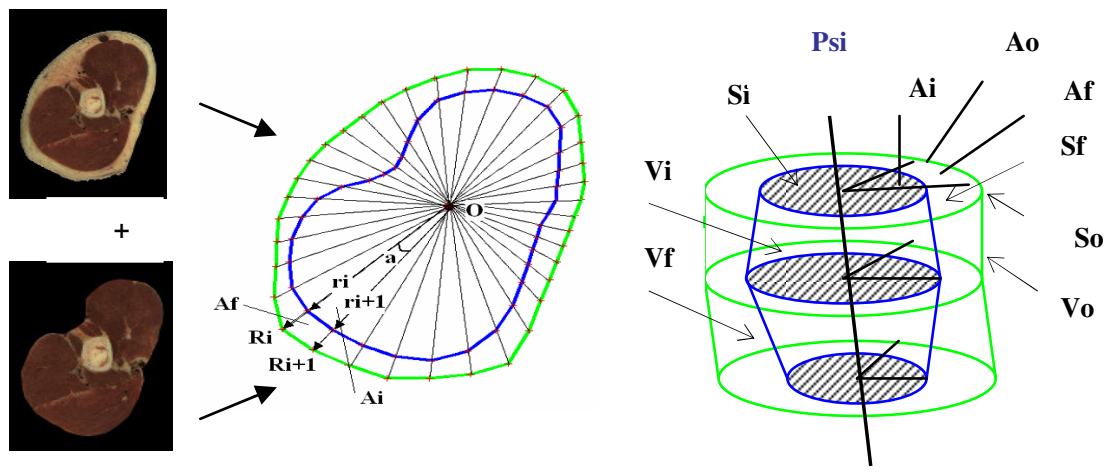

Fig. 4. (a) Ray casting each pair of cross section images (with and without fat layer) to retrieve the inner and outer radii for each sectional ray. (b) Digitise the template fat distribution.

As illustrated in Figure 4, we firstly execute "ray-casting" for each pair of cross section images (with and without the fat layer). Secondly, given the extracted inner and outer radii, the value of $A_{i}$ and $A_{o}$ (angular inner and outer area) and $S_{i}$ and $S_{o}$ (sectional inner and overall area) is computed. Thirdly, given the vertical distance inbetween each pair of cross-sections, the inner and overall volume $\left(V_{i}\right.$ and $\left.V_{o}\right)$ for each body part is calculated. Correspondingly, the fat value $A_{f}, S_{f}$ and $V_{f}$ is obtained. Next, to digitise the template fat distribution, we first use $P_{b}=V_{f} / V_{o}$ and $P_{s}=S_{f} / S_{o}$ to compute the fat percentages for each body part $\left(P_{b}\right)$ and its sample cross-sections $\left(P_{s l, \ldots}, P_{s n}\right)$. We then encode the sectional fat distribution features as: $D_{s i}=P_{s i} / P_{b}(\mathrm{i}=1$, $2, \ldots \mathrm{n})$, where $\mathrm{n}$ is equal to the number of cross sections for a certain body part. Given the sectional fat area $\left(S_{f}\right)$ and angular fat area $\left(A_{f l}, \ldots A_{f m}\right)$, the angular fat distribution is encoded as $D_{a i}=A_{f i} / S_{f}(\mathrm{i}=1,2, \ldots \mathrm{m})$, where $\mathrm{m}$ is equal to 36 and 40 for the limb and torso respectively. Next, to morph this anatomical model with new body part fat percentages, we assume that the new fat accumulates proportionally according to the original template fat distribution (preserving $D_{s i}$ and $D_{a i}$ unchanged). Then, the corresponding skin vertex positions can be updated through this fattening scheme. 


\subsection{Template Fat Percentage Manipulation}

On the completion of template fat distribution digitisation, we investigated a bodyfattening scheme, through which the 3D generic model can be 'physically' morphed when given a set of new fat percentage values of individual body parts. These new body part fat percentages are deduced from a user's figure sketch (see Section 5). We assume that the fat is distributed according to the original template fat distribution $\left(D_{s i} \text { and } D_{a i} \text { unchanged }\right)^{2}$, whilst the inner body stays relatively invariable ( $S_{i}$ and $A_{i}$ unchanged). Thus, given a new body part fat percentage $P_{b}$, the new sectional fat percentages $P_{s i}(\mathrm{i}=1,2, \ldots \mathrm{n})$ and angular fat areas $A_{f i}(\mathrm{i}=1,2, \ldots \mathrm{m})$ can be calculated. Then, preserving the inner radii $\left(r_{i}\right)$ value, the corresponding skin vertex positions can be updated when outer radii $\left(R_{i}\right)$ change.

\section{From 2D Raw Figure Sketch to 3D Human Body Model}

\subsection{On-line Curve Stroke Processing}

Freehand figure sketching is essentially rough and imprecise. It contains various "noises" - wiggly/overlapping strokes, missing figure contours, asymmetrical body parts, as shown in Figure 5(a).

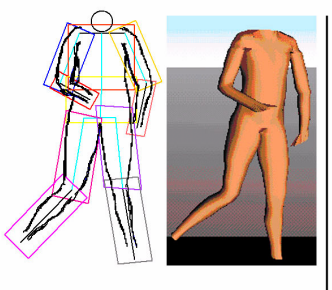

(a)

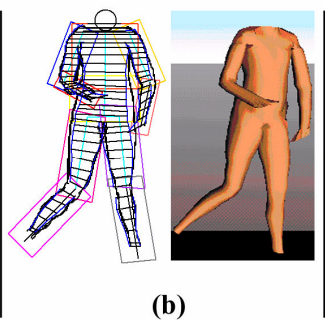

(b)

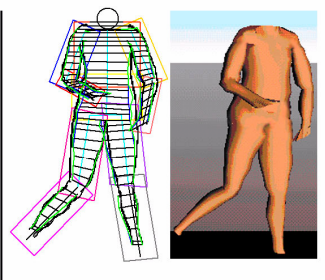

(c)

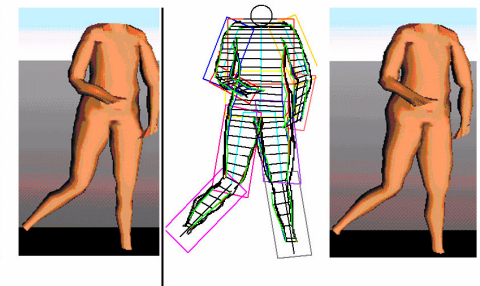

(d)

Fig. 5. (a) The input 2D freehand sketch and the 3D model after rigid morphing. (b) Graphical comparison to get the fat distribution measurements and the fatness morphed model (c) Graphical comparison to get the surface fitting measurements; the model with and without auto-beautification (d) Over-tracing body contour (right lower torso, left lower leg, and right leg) to modify an existing 3D surface model.

In this on-line drawing system, each curve stroke is processed (through stroke identification/segmentation) to identify its locating bone, given the pre-defined bone bounding boxes (see Figure 6(a)). The default bounding box sizes are determined heuristically based on the generic model proportion and a general body physique. Users can interactively adjust the bounding box sizes (e.g. when drawing a large human) and choose to show or hide the bounding boxes according to their needs. Then, this stroke is labeled (according to its assigned bone) and stored for later feature extraction use.

2 Everyone has a specific body fat distribution pattern, which is generally preserved when a body fattens or slims. In brief, the area with more fat is more likely to accumulate new fat, and vice versa. 


\subsection{Rigid Morphing and Body Feature Point Extraction}

Once the user completes a figure sketch and chooses to reconstruct the 3D model, a system automatic process starts for rigid morphing, fatness morphing and surface fitting. Rigid morphing is to deform the 3D generic model against the new body proportion and posture of the sketched figure. In our system, a "multi-layered backfront ambiguity clarifier" [10] is developed to recover the $3 \mathrm{D}$ pose from the $2 \mathrm{D}$ figure sketch. A skeleton-driven skin deformation technique [16] is applied for rigid morphing. After rigid morphing, the updated 3D template is projected onto $2 \mathrm{D}$ (Figure 5(b)) to obtain the body silhouette and 2D cross lines (projected from template cross-section slices). Then, indexed intersection points (template feature points) are found between 2D cross lines and the parsed template silhouette. We use Tf $\left[L f_{i j}, R f_{i j}\right]$ to save this template feature point set, where $L f_{i j}$ and $R f_{i j}$ represent the feature point lists on the left and right side of each body part. (i - body part index, $\mathrm{j}$ cross section index). Similarly, intersection points (sketch feature point set $S f\left[L f_{i j}\right.$, $\left.R f_{i j}{ }^{\prime}\right]$ ) are found between the saved sketch strokes and the template cross lines. Here, an average left/right feature point is taken if there are more than one intersection points extracted on either side of a body part (for each cross section) because of multiple rendering stokes.
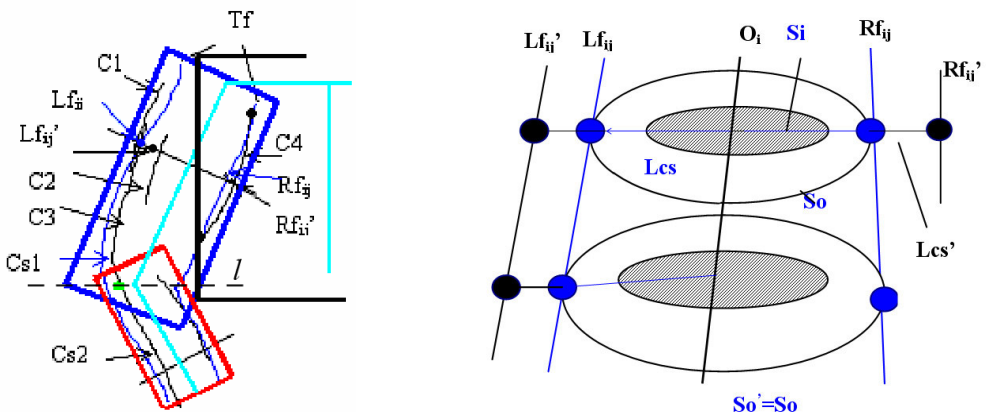

Fig. 6. (a) $2 \mathrm{D}$ raw sketch processing. For the multiple strokes $\mathrm{C} 1, \mathrm{C} 2$ and $\mathrm{C} 3$, an average point $\mathrm{Lf}_{\mathrm{ij}}$ ' is computed among the corresponding sectional intersection points. The stroke $\mathrm{C} 4$ is located in both the upper torso and upper arm bounding area. After "stroke identification", it is assigned to the upper arm due to a minimum in-between angle with the upper arm than the upper torso. A long stroke C3 is first segmented into stroke Cs1 and Cs2 (through "stroke segmentation"), which are further checked upon the locating bones. (b) Fat distribution estimation.

\subsection{Fat Distribution Estimation and Fatness Morphing}

The rigid-morphed 3D template model (see Figure 5(a)) is used to estimate the body part fat percentages of the 2D sketched figure. Here, we assume that when a body fattens/slims, its inner volume stays relatively unchanged compared with the fat volume. That is, for each body cross-section, only its overall area enlarges when new fat accumulates whilst its inner area stays invariant. In this sense, the fat percentage of each body part on a 2D figure drawing can be estimated through graphical comparison. As shown in Figure 6(b), since the template inner area $S_{i}$ and outer area $S_{o}$ are known for each cross-section, a new outer area $S_{o}$ ' can be calculated for the given cross section through $S_{o}{ }^{\prime}=S_{o} *\left(L_{c s}{ }^{\prime} / L_{c s}\right)$ (whilst $\left.S_{i}{ }^{\prime}=S_{i}\right) . L_{c s}$ ' and $L_{c s}$ here stand for the corresponding cross line lengths on the 2D template and sketched figure, which can be obtained by computing the distance between the previously extracted sketch feature points $\left(L f_{i j}, R f_{i j}{ }^{\prime}\right)$ and the template feature points $\left(L f_{i j}, R f_{i j}\right)$. For some 
cross-sections that have missing sketch points, $L_{c s}$ ' is set to be equal to $L_{c s}$. After getting the $S_{i}$ ' and $S_{o}$ ' for each cross section, a new body part fat percentage $P_{b}$ ' can be obtained as described in Section 4.2, given a set of known cross section in-between distances. Through this graphical comparison process, body part fat percentages across the whole body can be estimated for the 2D sketched figure. A fatnessmorphed model (see Figure 5(b)) can be achieved through deforming the rigidmorphed generic model according to the pre-defined body-fattening scheme (Section 4.3), given a set of new fat percentage input. As illustrated, after fatness morphing, 3D body is "biologically" transformed with fattened belly and thighs.

\subsection{Surface Fitting and Interactive 3D Model Modification}

After that, surface fitting is performed to match the $3 \mathrm{D}$ template with the $2 \mathrm{D}$ sketch. Here, an auto-beautification option is provided to regularise the 3D asymmetrical body from users' drawing imperfections (Figure 5(c)). After the above processing, users are given a realistic 3D character model from their freehand sketch. Then, they can interactively refine this $3 \mathrm{D}$ model by over-sketching its $2 \mathrm{D}$ figure profiles (Figure 5(d)). Modifications can be made at any time and on any key frame sketch to achieve the updated 3D model.

\subsection{Results and Discussion}

As shown in Fig. 7 and Fig 9(top), our sketch-based modelling interface interprets users' freehand figure sketches into plausible 3D human models of various body proportions, shapes, and postures. After illustrating a stick figure posture, users can flesh it out in flexible drawing sequences with natural and artistic contour strokes, like drawing on an electronic canvas. Figure 7 shows a series of freehand figure sketches (featured by multiple/overlapping strokes, body part foreshortening/occlusion, and drawing imperfections) and their 3D counterpart models in a mixture of figure postures (e.g. Fig. 7a/e - kneeling, Fig. 7b - picking, Fig. 7c - dancing, and Fig. 7d sitting), drawing views (e.g. Fig. 7a/d - side views, Fig. 7b/c/e - front views), and body shapes (varied body proportions and fat distribution). Our system can handle figure sketches with large overlying areas as shown in Fig. 7a, 7b, 7c, and 7e. Here, the occlusion order of the overlying body parts is solved by identifying the orientation of foreshortened body parts. The problem of missing body contours is handled by labeling the unfilled sketch feature points and processing them specifically during template morphing. Figure 9(top) shows a range of human body shapes (e.g. apple, pear, hour glass, etc, with distinct body sizes and fat distributions) and even a super human model (S8) generated by different users in a formal user test of our system.

Although our current human models are not as realistic as those generated from [1][5][6] for commercial use, we aimed to provide an intuitive and low-cost tool to enable non-professional users (2D artists and ordinary users) to sketch-out virtual humans and animation for design and entertaining purposes. Since people draw differently with varied drawing skills, the resulting 3D model should primarily match the original sketch as well as showing reasonably plausible effects to meet users' expectation. Therefore, a realistic 3D body model from a rough figure sketch may appear unnatural and may inhibit creativity. Moreover, our system is meant to be a fast prototyping machine for the early stage of virtual human modelling and 
animation. Therefore, the sketch-generated 3D characters and motions from our system can be exported to and refined by commercial tools to meet practical needs.
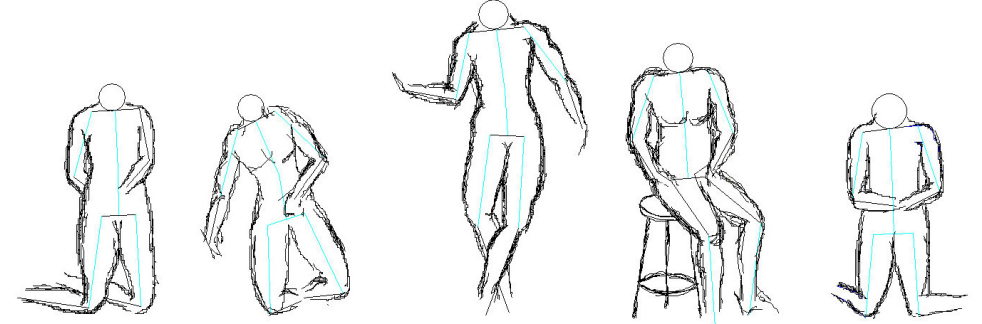

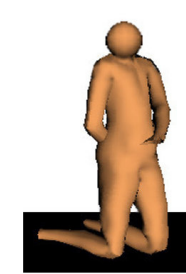

(a)

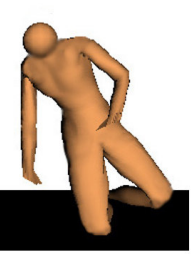

(b)

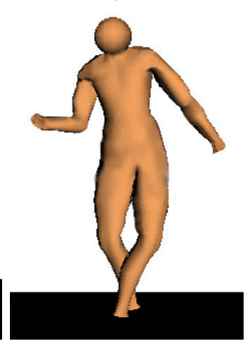

(c)

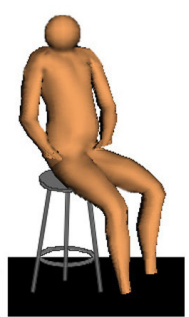

(d)

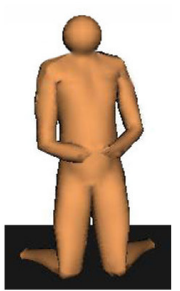

(e)

Fig. 7. Freehand 2D figure sketches and the reconstructed 3D human body models; the inner contours on some sketches (b, d) were added after 3D model generation, which shows the needs of depicting detailed surface shape through more rendering forms (e.g. suggestive contours).

\section{Creating 2D and 3D Virtual Human Animation}

Following the "Stick Figure $\rightarrow$ Fleshing-out $\rightarrow$ Skin Mapping" pipeline, a 3D virtual human animation is accomplished by wrapping the sketch-generated skin surface onto a series of posed stick figures, which can be further interpolated via VRML. Our system generates $2 \mathrm{D}$ silhouette/NPR animation by successively interpolating the extracted key figure contours (with the mapped fleshing-out contours) and playing their in-betweens on sketching interface. Figure 8 shows the snapshots of a 2D NPR animation in a doodled countryside view. Beside the single character animation, our system offers the opportunity to create group animation of a population of virtual humans and to storyboard intercommunications between virtual humans [17] (Fig 9).

\section{Implementation and User Experiences}

Our prototype modelling and animation system is implemented by Microsoft Visual $\mathrm{C}++$, MATLAB, and VRML. This system has been tested by various users (including artists, an animator, undergraduates and postgraduates, and a 12-year-old boy) through performance tests, sketching observation, and user interviews. After a short tutorial, users rapidly learned the modelling process, and began to sketch-out their own virtual human animation (on Tablet PC) within minutes (6.27 and 6.75 minutes 
on average for a three-frame stick figure and full figure animation respectively). Figure 9 (Top) shows the sketches and various human bodies created by users, which have been integrated into two group animations, shown in Figure 9 (Bottom).
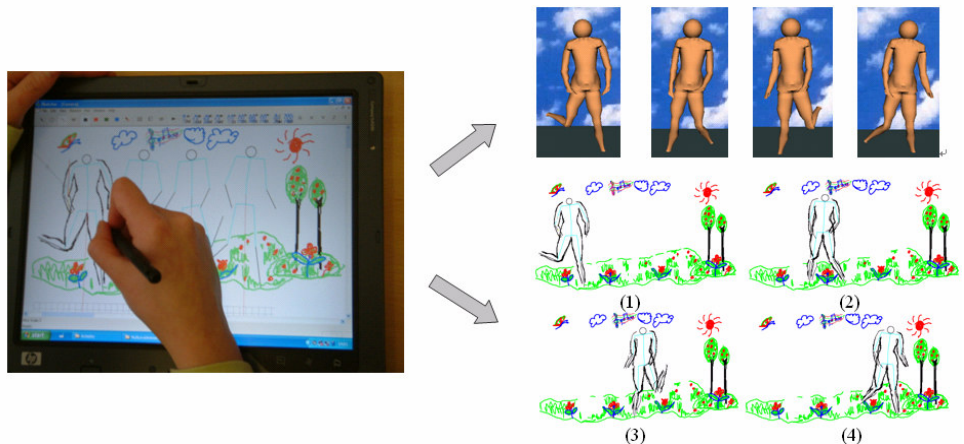

Fig. 8. (Left) The user is sketching-out a virtual human and its motion on a Tablet PC; (Top right) A sketch-generated 3D dancing character; (Bottom right) A 2D NPR animation played on the sketching interface in a doodled countryside view.
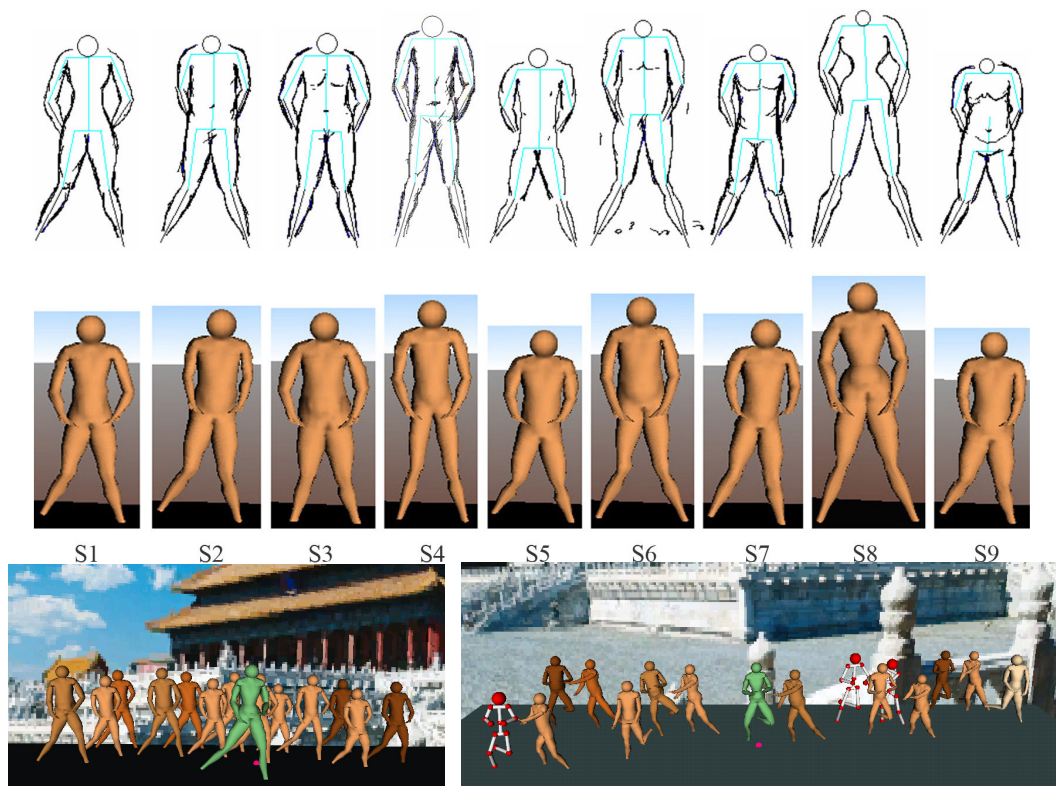

Fig. 9. (Top) A variety of 3D virtual humans and the original drawings created from different users: an artist (S3), a designer (S4), an animator (S6, S7), graduate students (S1, S2, S5, and S9), and a child (S8). (Bottom left) A population of user-created 3D virtual humans (skin colours varied by authors) are playing Chinese Kungfu together in a vivid 3D world with music (the pink ball on the ground is a music trigger). (Bottom right) A crowd of virtual humans and stick figures are fighting with each other in a $3 \mathrm{D}$ virtual world. Two of them (the $5^{\text {th }}$ from the left, and the furthest right figures) are performing different actions. It shows individuality among the collective behaviour. 


\section{Conclusion and Future Work}

In this paper, we have presented our new method and a smart interface for sketching out 3D virtual humans and animation. We investigated a "creative model-based method", which performs a human perception process to transfer 2D freehand sketches into 3D human bodies with various body sizes, shapes, and fat distributions. We created an anatomical three-layered model, which can be morphed against the "perceived" features of the original figure sketch. Our system is well designed for tolerating the abstraction, ambiguity, and imprecision of natural sketches. In the future, we are going to extend our generic template database to allow the generation of a wider range of virtual humans. We plan to improve our current 2D-to-3D modelling techniques to handle more complicated figure poses (e.g. lying) and views (e.g. top, back view). We propose to adopt more rendering forms including suggestive contours [18], shading, etc, to depict subtle surface features. Moreover, we intend to implement sketch-based modelling of human heads/hands/feet and clothes to deliver a more sophisticated character model.

\section{References}

1. Turner, R., Gobbetti, E.: Interactive Construction and Aanimation of Layered Elastically Deformable Characters, Computer Graphics Forum 17, 2 (1998) 135-152.

2. Choi, J. J.: MAYA Character Animation. $2^{\text {nd }}$ edition, San Francisco, Calif.: London: SYBEX (2004).

3. Fua, P.: Human Modelling from Video Sequence, Geometric Info Magazine 13, 7 (1999) 63-65.

4. Lee, W., Gu, J., and Magnenat-Thalmann, N.: Generating Animatable 3D Virtual Humans from Photographs, Computer Graphics Forum 19, 3 In Proc. Eurographics '2000 (2000) 1-10.

5. Anguelov, D., Srinivasan, P., Koller, D., Thrun, S., and Rodgers, J.: SCAPE: Shape Completion and Animation of People, Proc. SIGGRAPH 05 (2005) 408-416.

6. Seo, H., Magnenat-Thalmann, N.: An Example-based Approach to Human Body Manipulation, Graphical Models 66, 1 (2004) 1-23.

7. Igarashi, T., Matsuoka, S., Tanaka, H.: Teddy: A Sketching Interface for 3D Freeform Design. In Proc. SIGGRAPH'99 (1999) 409-416.

8. Karpenko, O., Hughes, J. F., Raskar, R: Free-form Sketching with Variational Implicit Surfaces, Proc. Eurographics 2002 21, 3 (2002)

9. Davis, J., Agrawala, M., Chuang, E., Popović , Z., and Salesin, D.: A Sketching Interface for Articulated Figure Animation. In Proc. Eurographics/ SIGGRAPH Symposium on Computer Animation (2003) 320-328

10. Mao, C, Qin, SF, Wright, DK: A Sketch-based Gesture Interface for Rough 3D Stick Figure Animation, Proc. of Eurographics Workshop on Sketch Based Interfaces and Modeling (2005) 175-183.

11. Thorne, M., Burke, D., van de Panne, M.: Motion Doodles: An Interface for Sketching Character Animation. ACM Transactions on Graphics (TOG) 23, 3 (2004) 424- 431.

12. Hoshino, J., Hoshino, Y.: Intelligent Storyboard for Prototyping Animation. In Proc. IEEE Int. Conf. On Multimedia and Expo (2001).

13. National Library of Medicine: The Virtual Human Project, http://www.nlm.nih.gov/research/visible/visiblehuman.html.

14. Lipson, H., Shpitalni, M.: Correlation-based Reconstruction of a 3D Object from a Single Freehand Sketch, In Proc. AAAI Spring Symposium Series - Sketch Understanding (2002).

15 EPA: Information on Whole Body Scanning, http://www.epa.nsw.gov.au/radiation/ctbodyscans.htm.

16. Weber J.: Run-time Skin Deformation, Intel Architecture Labs, http://www.intel.com/ial/3dsoftware/index.htm (2006).

17. Mao, C., Qin, S.F., Wright, D.K., Sketching-out Virtual Humans: from 2D Storyboarding to Immediate 3D Character Animation, Proc.of ACM SIGCHI International Conference on Advances in Computer Entertainment Technology, Hollywood, Los Angeles, (2006).

18. DeCarlo, D., Finkelstein, A., Rusinkiewics, S. and Santella, A.: Suggestive Contours for Conveying Shape, ACM Transaction on Graphics 22, 3 (2003) 848-855. 